\title{
STRONG LAWS OF LARGE NUMBERS FOR ARRAYS OF ROWWISE INDEPENDENT RANDOM ELEMENTS
}

\author{
ROBERT LEE TAYLOR \\ Department of Statistics \\ University of Georgia \\ Athens, GA 30602 U.S.A. \\ TIEN-CHUNG HU \\ Department of Mathematics \\ National Tsing-Hua University \\ Hsin-chu, Taiwan R.O.C. \\ (Received November 18, 1986)
}

ABSTKACT. Let $\left\{\mathrm{X}_{\mathrm{nk}}\right\}$ be an array of rowwise independent random elements in a separable Banach space of type $\mathrm{p}+\delta$ with $\mathrm{EX}_{\mathrm{nk}}=0$ for all $\mathrm{k}, \mathrm{n}$. The complete convergence (and hence almost sure convergence) of $n^{-1 / p} \sum_{k=1}^{n} x_{n k}$ to $0,1 \leq p<2$, is obtained when $\left\{\mathrm{X}_{\mathrm{nk}}\right\}$ are uniformly bounded by a random variable $\mathrm{X}$ with $\mathrm{E}|\mathrm{X}|^{2 \mathrm{p}}<\infty$. When the array $\left\{\mathrm{X}_{\mathrm{nk}}\right\}$ consists of i.i.d. random elements, then it is shown that $\mathrm{n}^{-1 / \mathrm{P}_{\sum}} \mathrm{n}_{\mathrm{k}=1} \mathrm{X}_{\mathrm{nk}}$ converges completely to 0 if and only if $\mathrm{E}\left\|\mathrm{X}_{11}\right\|^{2 \mathrm{p}}<\infty$.

KEY WORDS AND PHRASES. Random elements, Strong laws of large numbers, Complete Convergence, Rademacher type $\mathrm{p}+\delta$ spaces.

1980 MATHEMATICS SUBJECT CLASSIFICATION CODE. $60 \mathrm{~B} 12$

\section{INTRODUCTION AND PRELIMINARIES.}

Let $(E,\|\|)$ be a real separable Banach space. Let $(\Omega, A, P)$ denote a probability space. A random element $X$ in $E$ is a function from $\Omega$ into $E$ which is $A$-measurable with respect to the Borel subsets $B(E)$. The $\mathrm{p}^{\text {th }}$ absolute moment of a random element $X$ is $E\|X\|^{p}$ where $E$ is the expected value of the random variable $\|X\|^{p}$. The expected value of $X$ is defined to be the Bochner integral (when $E\|X\|<\infty$ ) and is denoted by EX. The concepts of independence and identical distributions have direct extensions to $E$. A separable Banach space is said to be of (Rademacher) type $p, 1 \leq p \leq 2$, if there exists a constant $C$ such that

$$
E\left\|\sum_{k=1}^{n} X_{k}\right\|^{p} \leq C \sum_{k=1}^{n} E\left\|X_{k}\right\|^{p}
$$

for all independent random elements $x_{1}, \ldots, x_{n}$ with zero means and finite $p^{\text {th }}$ moments. Every separable Hilbert space and finite-dimensional Banach space is of type 2 . Every separable Banach space is at least type 1 while the $\ell^{\mathrm{P}}$ and $\mathrm{L}^{\mathrm{P}}$ spaces are of type $\min \{2, \mathrm{p}\}$ for $\mathrm{p} \geq 1$.

Throughout this paper $\left\{\mathrm{X}_{\mathrm{nk}}\right.$ : $\left.1 \leq \mathrm{k} \leq \mathrm{n}, \mathrm{n} \geq 1\right\}$ will denote rowwise independent random elements in $E$ such that

$$
\mathrm{EX}_{\mathrm{nk}}=0 \cdot \text { for } \mathrm{all} \mathrm{n} \text { and } \mathrm{k}
$$

and such that $\left\{\mathrm{X}_{\mathrm{nk}}\right\}$ are uniformly bounded by a random variable $\mathrm{X}$ with

$$
\mathrm{E}|\mathrm{X}|^{2 \mathrm{p}}<\infty \quad \text { for some } 1 \leq \mathrm{p}<2 \text {. }
$$


Recall that an array $\left\{\lambda_{\mathrm{nk}}\right\}$ of random elenents is said to be uniformly bounded by a random variable $X$ if for $a l l n$ and $k$ and for every real number $t>0$

$$
P\left[\left\|x_{n k}\right\|>t\right] \leq P[|X|>t] \text {. }
$$

Note that i.i.d. random elements are uniformly bounded by $\left\|\mathrm{X}_{11}\right\|$. The major results of this paper show that

$$
\frac{1}{\mathrm{n}^{1 / \mathrm{p}}} \sum_{\mathrm{k}=1}^{\mathrm{n}} \mathrm{x}_{\mathrm{nk}} \rightarrow 0 \text { completely }
$$

where complete convergence is defined (as in Hsu and Robbins [1]) by

$$
\sum_{n=1}^{\infty} P\left[\left\|\frac{1}{n^{1 / p}} \sum_{k=1}^{n} X_{n k}\right\|>\varepsilon\right]<\infty
$$

for each $\varepsilon>0$.

Erdös [2] showed that for an array of i.i.d. random variables $\left\{\mathrm{x}_{\mathrm{nk}}\right\},(1.4)$ holds if and only if $\mathrm{E}\left|\mathrm{x}_{11}\right|^{2 \mathrm{p}}<\infty$. Jain [3] obtained a uniform strong law of large numbers for sequences of i.i.d. random elements in separable Banach spaces of type 2 which would yield (1.4) with $p=1$ for an array of i.i.d. random elements $\left\{\mathrm{X}_{\mathrm{nk}}\right\}$ in a type 2 space. Woyczynski [4] showed that

$$
\frac{1}{n^{1 / p}} \sum_{k=1}^{n} x_{k} \rightarrow 0 \text { completely }
$$

for any sequence $\left\{x_{n}\right\}$ of independent random elements in a type $p+\delta, 1 \leq p<2$ and $\delta>0$, with $\operatorname{EX}_{\mathrm{n}}=0$ for all $\mathrm{n}$ which is uniformly bounded by a random variable $\mathrm{X}$ satisfying $\mathrm{E}|\mathrm{X}|^{\mathrm{P}}<\infty$. Móricz, Hu and Taylor [5] showed that Erdös' result could be obtained by replacing the i.i.d. condition by the uniformly bounded condition (1.3). In addition, they showed that Jain's result for i.i.d. random elements with $p=1$ did not require the space to be type 2 but held in all separable Banach spaces. In this paper, (1.4) is established in type $p+\delta$ spaces, $1 \leq p<2$ and $\delta>0$, for uniformly bounded rowwise independent random elements. For i.i.d. random elements in type $p+\delta$ spaces, it is shown that (1.4) holds if and only if $\mathrm{E}\left\|\mathrm{X}_{11}\right\|^{2 \mathrm{p}}<\infty$. Thus, no sharper moment conditions are possible.

\section{MAJOR RESULTS.}

Many authors (starting with Beck [6]) have related the strong law of large numbers for non-identically distributed, independent random elements in separable Banach spaces to the necessity of the space being of type $p+\delta$ for $1 \leq p<2$ and some $\delta>0$. Consequently, attention is restricted to type $p+\delta$ spaces in this paper. Three lemmas will be used in obtaining the major results. They are stated here without proof. Lemma 1 with $r=1$ is in most textbooks while Lemma 2 is accomplished using integration by parts. Lemma 3 is in Woyczynski [4].

LEMMA 1. For any $r \geq 1, E|X|^{r}<\infty$ if and only if

$$
\sum_{n=1}^{\infty} n^{r-1} P[|x|>n]<\infty \text {. }
$$

More precisely, $r 2^{-r} \sum_{n=1}^{\infty} n^{r-1} P[|x|>n]$

$$
\leq \mathrm{E}|\mathrm{X}|^{\mathrm{r}} \leq 1+\mathrm{r} 2^{\mathrm{r}} \sum_{\mathrm{n}=1}^{\infty} \mathrm{n}^{\mathrm{r}-1} \mathrm{P}[|\mathrm{X}|>\mathrm{n}] \text {. }
$$


LEMMA 2. If $r \geq 1$, then for any $p>0$

$$
E\left(|X|^{r} I\left[|x| \leq n^{1 / p}\right] \leq r \int_{0}^{n^{1 / p}} t^{r-1} P[|x|>t] d t\right.
$$

and

$$
E\left(|X| I_{\left[|X|>n^{1 / p}\right]}\right)=n^{1 / p_{P}}\left[|X|>n^{1 / p}\right]+\int_{n}^{\infty} P[|x|>t] d t .
$$

LEMMA 3. Let $1 \leq \mathrm{p} \leq 2$ and $\mathrm{q} \geq 1$. The following properties are equivalent:

(i) $E$ is of type p.

(ii) There exists a $C$ such that for all independent random elements $x_{1}, \ldots, x_{n}$ in $E$ with $\mathrm{EX}_{\mathrm{k}}=0, \mathrm{k}=1, \ldots, \mathrm{n}$,

$$
E\left\|\sum_{k=1}^{n} x_{k}\right\|^{q} \leq C E\left(\sum_{k=1}^{n}\left\|x_{k}\right\|^{p}\right)^{q / p} \text {. }
$$

THEOREM 4. If $\left\{\mathrm{X}_{\mathrm{nk}}\right\}$ is an array of rowwise independent random elements in a type $\mathrm{p}+\delta$ space, $1 \leq \mathrm{p}<2$ and $\delta>0$, which are uniformly bounded by a random variable $X$ such that (1.1) and (1.2) holds, then

$$
\frac{1}{n^{1 / p}} \sum_{k=1}^{n} X_{n k} \rightarrow 0 \text { completely. }
$$

PROOF. Define

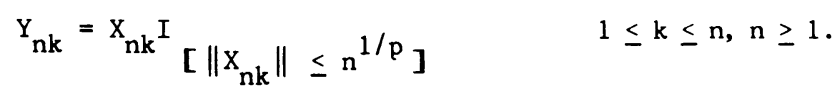

Then, by Lemma 1 (with $r=2$ ),

$$
\begin{aligned}
& \sum_{n=1}^{\infty} \sum_{k=1}^{n} P\left[x_{n k} \neq Y_{n k}\right]=\sum_{n=1}^{\infty} \sum_{k=1}^{n} P\left[\left\|x_{n k}\right\|>n^{1 / P}\right] \\
& \leq \sum_{n=1}^{\infty} n P\left[|x|>n^{1 / p}\right] \\
& =\sum_{n=1}^{\infty} n P\left[|x|^{P}>n\right] \leq 2 E|x|^{2 P}<\infty .
\end{aligned}
$$

Next, for any $\varepsilon>0$,

$$
\begin{aligned}
& \sum_{n=1}^{\infty} P\left[\left\|\frac{1}{n} \sum_{k=1}^{n} X_{n k}-\frac{1}{n^{1 / p}} \sum_{k=1}^{n} Y_{n k}\right\|>\varepsilon\right] \\
& \leq \sum_{n=1}^{\infty} P\left[\sum_{k=1}^{n}\left[X_{n k} \neq Y_{n k}\right]\right] \\
& \leq \sum_{n=1}^{\infty} \sum_{k=1}^{n} P\left[X_{n k} \neq Y_{n k}\right]<\infty .
\end{aligned}
$$

Therefore,

$$
\left\|\frac{1}{n^{1 / p}} \sum_{k=1}^{n} X_{n k}-\frac{1}{n^{1 / p}} \sum_{k=1}^{n} Y_{n k}\right\| \rightarrow 0 \text { completely, }
$$

and it sufficies to prove that

$$
\left\|\frac{1}{n^{1 / p}} \sum_{k=1}^{n} Y_{n k}\right\| \rightarrow 0 \text { completely. }
$$


To this end, let

$$
Z_{n k}=Y_{n k}-E Y_{n k} \quad(k=1,2, \ldots, n ; n=1,2, \ldots) .
$$

Then for $1 \leq q \leq 2 p$ it follows by Hölder's inequality that

$$
\begin{aligned}
& \left(E\left\|Z_{n k}\right\|^{q}\right)^{1 / q} \leq 2\left(E\left\|Y_{n k}\right\|^{q}\right)^{1 / q} \\
& \leq 2\left(E\left\|Y_{n k}\right\|^{2 p}\right)^{1 /(2 p)} \leq 2\left(E|X|^{2 p}\right)^{1 /(2 p)},
\end{aligned}
$$

so that

$$
\begin{aligned}
& E\left\|z_{n k}\right\|^{q} \leq 2^{q}\left(E|X|^{2 p}\right)^{q /(2 p)} \\
& \leq 2^{2 p}\left(1+E|X|^{2 p}\right)=C_{1} \text {, say. }
\end{aligned}
$$

Furthermore,

$$
\left\|Z_{n k}\right\| \leq\left|Y_{n k}\left\|+\mid E Y_{n k}\right\| \leq 2 n^{1 / p}\right.
$$

Following the techniques of Taylor [7] in expanding a high power of a sum, let $\mathrm{r}=\mathrm{p}+\delta$ and $v$ be chosen so that

$$
\mathrm{s}=\frac{v}{\mathrm{r}} \text { is an integer and } v>\left(\frac{1}{\mathrm{p}}-\frac{1}{\mathrm{r}}\right)^{-1} .
$$

It is readily seen that $\mathrm{E}\left(\sum_{\mathrm{k}=1}^{\mathrm{n}}\left\|\mathrm{Z}_{\mathrm{nk}}\right\|\right)^{\nu}<\infty$, so that, by Lemma 3 ,

$$
\begin{aligned}
E\left(\left\|\sum_{k=1}^{n} z_{n k}\right\|\right)^{\nu} & \leq C E\left(\sum_{k=1}^{n}\left\|Z_{n k}\right\|^{r}\right)^{s} . \\
& =C k_{1}, \ldots, k_{s} E\left(\prod_{j=1}^{s}\left\|z_{n k_{j}}\right\|^{r}\right)
\end{aligned}
$$

where the sum is extened for all s-tuples $\left(k_{1}, \ldots, k_{s}\right)$ with $k_{j}=1,2, \ldots, n$ for each $j$. The general term to be considered then will have

where

$$
\begin{gathered}
q_{1} \text { of the } k^{\prime} s=\xi_{1}, \ldots, q_{m} \text { of the } k^{\prime} s=\xi_{m} ; \\
r_{1} \text { of the } k^{\prime} s=\eta_{1}, \ldots, r_{\ell} \text { of the } k^{\prime} s=\eta_{l} ; \\
r \leq r q_{i} \leq 2 p, r r_{j}>2 p, \text { and } \\
\sum_{i=1}^{m} q_{i}+\sum_{j=1}^{\ell} r_{j}=s .
\end{gathered}
$$

Clearly, $q_{i}=1$. Then, using (2.3) and (2.4), we can conclude that

$$
\begin{aligned}
& E\left(\prod_{i=1}^{m}\left\|z_{n \xi_{i}}\right\|^{r q_{i}} \prod_{j=1}^{\ell}\left\|z_{n n_{j}}\right\|^{r r_{j}}\right) \\
& =\prod_{i=1}^{m} E\left\|z_{n \xi_{i}}\right\|^{r q_{i}} \prod_{j=1}^{\ell} E\left(\left\|z_{n \eta_{j}}\right\|^{2 p}\left\|z_{n \eta_{j}}\right\|^{r r_{j}-2 p}\right) \\
& \leq C_{1}^{m+\ell} \prod_{j=1}^{\ell}\left(2 n^{1 / p}\right)^{r r_{j}-2 p} \\
& =C_{1}^{m+\ell} \sum_{j=1}^{\ell}\left(r r_{j}-2 p\right) \sum_{n^{j=1}}^{\ell}\left(r r_{j} / p\right)-2 \ell
\end{aligned}
$$




$$
\begin{aligned}
& \leq C_{1}^{\nu} 2^{\nu} n^{\sum_{j=1}^{x}\left(r r_{j} / p\right)-2 \ell} \\
& =C_{2} n^{\sum_{j=1}^{\ell}\left(r r_{j} / p\right)-2 \ell} \text {, say. }
\end{aligned}
$$

Combining all possible terms of form (2.9), we can write

$$
\begin{aligned}
& E\left(\sum_{k=1}^{n}\left\|z_{n k}\right\|^{r}\right)^{s}
\end{aligned}
$$

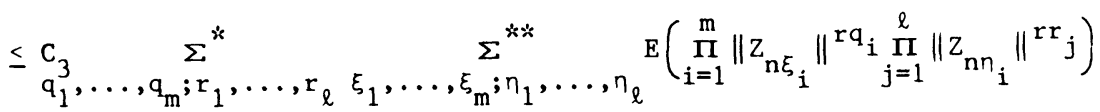

$$
\begin{aligned}
& =\mathrm{C}_{3}, \ldots, \mathrm{q}^{*} ; \mathrm{r}_{1}, \ldots, \mathrm{r}_{\ell} \quad \mathrm{s} \mathrm{q}_{1}, \ldots, \mathrm{q}_{\mathrm{m}} ; \mathrm{r}_{1}, \ldots, \mathrm{r}_{\ell} \text {, say, }
\end{aligned}
$$

where $\Sigma^{*}$ is extended over all m-tuples $\left(q_{1}, \ldots, q_{m}\right)$ and $\ell$-tuples $\left(r_{1}, \ldots, r_{\ell}\right)$ such that Conditions (2.7) and (2.8) are satisfied (the cases $m=0$ or $\ell=0$ may also occur), while $\Sigma^{* *}$ is extended over all $(m+\ell)$ - tuples $\left(\xi_{1}, \ldots, \xi_{m} ; \eta_{1}, \ldots, \eta_{\ell}\right)$ of different integers between 1 and $\mathrm{n}$ and $\mathrm{C}$ is a constant independent of $\mathrm{n}$. Let $\mathrm{m}+\ell=t$ Obviously, $1 \leq t \leq s$. We distinguish two cases according to $t \geq 2$ or $t=1$.

Case $t \geq 2$. By (2.9)

$$
\begin{aligned}
& \mathrm{s}_{\mathrm{q}_{1}, \ldots, \mathrm{q}_{\mathrm{m}} ; \mathrm{r}_{1}, \ldots, \mathrm{r}_{\ell}} \\
& \leq \mathrm{C}_{2} \xi_{1}, \ldots, \xi_{m} ; \eta_{1}, \ldots, \eta_{\ell} \mathrm{n}^{\sum_{j=1}^{\ell}\left(\mathrm{rr}_{j} / \mathrm{p}\right)-2 \ell} \\
& \leq \mathrm{C}_{2} \mathrm{n} \sum_{j=1}^{\ell}\left(\mathrm{rr}_{j} / \mathrm{p}\right)-2 \ell+t
\end{aligned}
$$

Now, the power to which $\mathrm{n}$ is raised here can be estimated by means of $(2.8)$ and $q_{i}=1$ as follows

$$
\begin{aligned}
& \frac{1}{p} \sum_{j=1}^{\ell} r r_{j}-2 \ell+t \\
= & \frac{1}{p}\left(r s-\sum_{i=1}^{m} r_{i}\right)-2(t-m)+t \\
= & \frac{v}{p}-\frac{r m}{p}-2(t-m)+t \\
= & \frac{v}{p}-t-m\left(\frac{r}{p}-2\right) .
\end{aligned}
$$

We distinguish two further subcases according to $m=t$ or $m \leq t-1$.

Subcase $m=t$. By assumption $1 \leq p<2$. Also, $q_{i}=1$ for each $i$.

Thus, $\mathrm{m}=\mathrm{s}$ and, by $(2.5)$. 


$$
\begin{aligned}
t+m\left(\frac{r}{p}-2\right) & =s\left(\frac{r}{p}-1\right) \\
& =v\left(\frac{1}{p}-\frac{1}{r}\right)>1 .
\end{aligned}
$$

Subcase $m \leq t-1$. Then $t-m \geq 1$ and even $t-m \geq 2$ in the particular case where $\mathrm{m}=0$. Thus, again

$$
t+m\left(\frac{r}{p}-2\right)=(t-m)+m\left(\frac{r}{p}-1\right)>1 .
$$

Now we turn to

Case $t=1$. In this case necessarily $m=0$ and $\ell=1$, consequently $r_{1}=s$ and

$$
s_{a_{1}, \ldots, q_{m} ; r_{1}, \ldots, r_{\ell}}=s_{0 ; s}=\sum_{k=1}^{n} E\left\|z_{n k}\right\|^{r s} \text {. }
$$

Using Lemma 2, we obtain that

$$
\begin{aligned}
& \sum_{1}=\sum_{n=1}^{\infty} \frac{1}{n^{\nu / p}} \sum_{k=1}^{n} E\left\|Z_{n k}\right\|^{\nu} \\
\leq & 2^{\nu} \sum_{n=1}^{\infty} \frac{1}{n^{\nu / p}} \sum_{k=1}^{n} E\left\|Y_{n k}\right\|^{\nu} \\
\leq & 2^{\nu} \sum_{n=1}^{\infty} \frac{1}{n^{\nu / p}} \sum_{k=1}^{n} \nu \int_{0}^{n^{1 / p}} t^{\nu-1} P\left[\left\|x_{n k}\right\|>t\right] d t \\
\leq & 2^{\nu} \sum_{n=1}^{\infty} \frac{1}{n^{\nu / p}} \nu n \int_{0}^{n^{1 / p}} t^{\nu-1} P[|x|>t] d t .
\end{aligned}
$$

Letting $t=n^{1 / p} s^{1 / v}$ and applying Lemma 1 (with $r=2$ ), it follows that

$$
\begin{aligned}
& \Sigma_{1} \leq 2^{\nu} \sum_{n=1}^{\infty} n \int_{0}^{1} P\left[|X|>n^{1 / p} s^{1 / \nu}\right] d s \\
= & 2^{\nu} \int_{0}^{1} \sum_{n=1}^{\infty} n P\left[\left|s^{-1 / \nu} x\right|^{p}>n\right] d s \\
\leq & 2^{v+1} \int_{0}^{1} s^{-2 p / \nu} E|x|^{2 p} d s \\
= & 2^{v+1} \frac{v}{v-2 p} E|x|^{2 p}<\infty .
\end{aligned}
$$

Using Markov's inequality, (2.7) and (2.10) - (2.15) we have, for any $\varepsilon>0$,

$$
\begin{aligned}
\Sigma_{2}(\varepsilon) & =\sum_{n=1}^{\infty} P\left[\left\|\frac{1}{n^{1 / p}} \sum_{k=1}^{n} z_{n k}\right\|>\varepsilon\right] \\
& \leq \sum_{n=1}^{\infty} \frac{1}{\left(\varepsilon n^{1 / p}\right)^{v}} E\left(\left\|\sum_{k=1}^{n} z_{n k}\right\|\right)^{\nu} \\
& \leq \sum_{n=1}^{\infty} \frac{C}{\left(\varepsilon n^{1 / p}\right)^{v}} E\left(\sum_{k=1}^{n}\left\|z_{n k}\right\|^{r}\right)^{s}
\end{aligned}
$$




$$
\begin{aligned}
& \leq \frac{C C_{3}}{\varepsilon^{\nu}}\left[\sum_{n=1}^{\infty} \frac{1}{n^{\nu / p}} \sum_{k=1}^{n} E\left\|Z_{n k}\right\|^{\nu}\right. \\
& \left.+\sum_{n=1}^{\infty} \frac{C_{2}}{n^{\nu / p}} \sum_{t=2}^{s} q_{1}, \ldots, q_{m} ; r_{1}, \ldots, r_{\ell} n^{\frac{\nu}{p}-t-m\left(\frac{r}{p}-2\right)}\right] \\
& =\frac{C C_{3}}{\varepsilon^{\nu}}\left[\sum_{1}+C_{2} \sum_{t=2}^{s} q_{1}, \ldots, q_{m} ; r_{1}, \ldots, r_{\ell} \sum_{n=1}^{\infty n}-t-m\left(\frac{r}{p}-2\right)\right],
\end{aligned}
$$

where $\Sigma^{(t)}$ means that the sum is extended over all m-tuples $\left(q_{1}, \ldots, q_{m}\right)$ and $\ell$-tuples $\left(r_{1}, \ldots, r_{\ell}\right)$ with Conditions $(2.7)$ and (2.8) such that $m+\ell=t$. Since the number of terms in each of $\Sigma^{(t)}$ is finite and the exponent of $n$ is less than -1 , for every $\varepsilon>0$, we have $\Sigma_{2}(\varepsilon)<\infty$. Thus, we have proved that

$$
\left\|\frac{1}{n^{1 / p}} \sum_{k=1}^{n} z_{n k}\right\| \rightarrow 0 \text { completely }(n \rightarrow \infty) \text {. }
$$

In order to prove $(2.2)$, we need to establish

$$
\Sigma_{3}=\sum_{n=1}^{\infty} \frac{1}{n^{1 / p}} \sum_{k=1}^{n}\left\|E Y_{n k}\right\|<\infty
$$

To achieve this goal, we will proceed as follows. By (2.1),

$$
\begin{aligned}
Y_{n k} & =X_{n k} I\left[\left\|x_{n k}\right\| \leq n^{1 / p}\right] \\
& =x_{n k}-X_{n k} I\left[\left\|x_{n k}\right\|>n^{1 / p}\right] .
\end{aligned}
$$

Since $\mathrm{EX}_{\mathrm{nk}}=0$, hence

$$
\left\|E Y_{n k}\right\| \leq E\left(\left\|X_{n k}\right\| I_{\left[\left\|X_{n k}\right\|>n^{1 / p}\right]}\right)
$$

Thus, using Lemma 2,

$$
\begin{aligned}
& \sum_{3} \leq \sum_{n=1}^{\infty} \frac{1}{n^{1 / p}} \sum_{k=1}^{n} E\left(\left\|x_{n k}\right\| I\left[\quad\left[x_{n k} \|>n^{1 / p}\right]\right)\right. \\
= & \sum_{n=1}^{\infty} \frac{1}{n^{1 / p}} \sum_{k=1}^{n}\left(n^{1 / p} P\left[\left\|x_{n k}\right\|>n^{1 / p}\right]+\int_{n}^{\infty} P\left[\left\|x_{n k}\right\|>t\right] d t\right) \\
\leq & \sum_{n=1}^{\infty}\left(n P\left[|x|>n^{1 / p}\right]+\frac{n}{n^{1 / p}} \int_{n^{1 / p}}^{\infty} P[|x|>t] d t\right) .
\end{aligned}
$$

Letting $t=n^{1 / p} s$ and applying Lemma 1, we can conclude that

$$
\begin{aligned}
& \Sigma_{3} \leq \sum_{n=1}^{\infty} n P\left[|X|^{p}>n\right]+\sum_{n=1}^{\infty} n \int_{1}^{\infty} P\left[|x|>n^{1 / p} s\right] d s \\
& \leq 2 E|X|^{2 p}+\int_{1}^{\infty} \sum_{n=1}^{\infty} P\left[\left|s^{-1} x\right|^{p}>n\right] d s
\end{aligned}
$$




$$
\begin{aligned}
& \leq 2 \mathrm{E}|\mathrm{X}|^{2 \mathrm{p}}+\int_{1}^{\infty} \mathrm{s}^{-2 \mathrm{p}} \mathrm{E}|\mathrm{X}|^{2 \mathrm{p}} \mathrm{ds} \\
& =\frac{4 \mathrm{p}-1}{2 \mathrm{p}-1} \mathrm{E}|\mathrm{X}|^{2 \mathrm{p}}<\infty
\end{aligned}
$$

proving (2.2) through (2.16), and thereby completing the proof of Theorem 4 .

Note that if $\sup _{n k} E\left\|X_{n k}\right\|^{2 p+\alpha}<\infty$, for some $\alpha>0$, then there exists a r.v. $X$ such that $\left\{\mathrm{X}_{\mathrm{nk}}\right\}$ are uniformly bounded by $\mathrm{X}$ and $\mathrm{E}|\mathrm{X}|^{2 \mathrm{p}}<\infty$. Therefore, Corollary 5 follows.

COROLLARY 5. Let $E$ be a type $p+\delta$ separable Banach space for $1 \leq p<2$ and $\delta>0$. If $\sup _{n k} E\left\|X_{n k}\right\|^{2 p+\alpha}<\infty$ for some $\alpha>0$, then

$$
\left\|\frac{1}{n^{1 / p}} \sum_{k=1}^{n} x_{n k}\right\| \rightarrow 0 \text { completely. }
$$

For type $1+\delta$ spaces, Taylor [7] obtained

$$
\sum_{k=1}^{\infty} a_{n k} x_{n k} \rightarrow 0 \text { completely }
$$

where $\left\{X_{n k}\right\}$ is uniformly bounded by X with $E|X|^{1+1 / r}<\infty$ and $\left\{a_{n k}\right\}$ are Toeplitz weights with $\max _{k}\left|a_{n k}\right|=O\left(n^{-r}\right)$. In the special case of uniform weights $a_{n k}=\frac{1}{n}, 1 \leq k \leq n$, then $r=1$ and Theorem 4 can be thought of as an extension of this result. Extension of Theorem 4 to infinite arrays and general weights $\left\{a_{n k}\right\}$ are possible but the detailed verification of their proofs are not included here. However, it will be shown next that the moment condition $\mathrm{E}|\mathrm{X}|^{2 \mathrm{P}}<\infty$ cannot be reduced in Theorem 4 . In particular, for an array $\left\{X_{n k}\right\}$ of i.i.d. random elements in a type $p+\delta$ space with $E_{11}=0$, it will be shown that the SLLN holds if and only if $\mathrm{E}\left\|\mathrm{X}_{11}\right\|^{2 \mathrm{p}}<\infty$.

THEOREM 6. Let $\left\{\mathrm{X}_{\mathrm{nk}}\right\}$ be an array of i.i.d. random elements in a type $\mathrm{p}+\delta$ space, $1 \leq \mathrm{p}<2$ and $\delta>0$, with $\mathrm{EX}_{11}=0$. Then $\mathrm{E}\left\|\mathrm{X}_{11}\right\|^{2 \mathrm{p}}<\infty$ if and only if

$$
\frac{1}{n^{1 / p}} \sum_{k=1}^{n} x_{n k} \rightarrow 0 \text { completely. }
$$

PROOF: From Theorem 4, we know that $E\left\|X_{11}\right\|^{2 p}<\infty$ implies (2.18) since the array $\left\{\mathrm{X}_{\mathrm{nk}}\right\}$ is uniformly bounded by $\left\|\mathrm{X}_{11}\right\|$.

Now, assume that (2.18) holds. Since $\left\{\mathrm{X}_{n k}\right\}$ are i.i.d., for every $\mathrm{n}$ and $\varepsilon>0$

$$
P\left[\left\|\frac{1}{n} \sum_{k=1}^{n} X_{k k}\right\|>\varepsilon\right]=P\left[\left\|\frac{1}{n / p} \sum_{k=1}^{n} X_{n k}\right\|>\varepsilon\right] \text {. }
$$

By (2.18), for every $\varepsilon>0$,

$$
\sum_{n=1}^{\infty} P\left[\left\|\frac{1}{n^{1 / P}} \sum_{k=1}^{n} X_{k k}\right\|>\varepsilon\right]<\infty,
$$

which says $\frac{1}{n^{1 / p}} \sum_{k=1}^{n} x_{k k} \rightarrow 0$ a.s.

As a consequence, 


$$
\frac{1}{n^{1 / p}} x_{n n}=\frac{1}{n^{1 / p}} \sum_{k=1}^{n} x_{k k}-\left(\frac{n-1}{n} \frac{1}{n-1}\right)^{1 / p} \sum_{k=1}^{n-1} x_{k k} \rightarrow 0 \text { a.s.. }
$$

Let $\varepsilon=1$. It follows from Lemma 1 (with $r=1$ ) and the Borel-Cantelli lemma that

$$
\begin{aligned}
E\left\|X_{11}\right\|^{p} & \leq 1+2 \sum_{n=1}^{\infty} P\left[\left\|X_{11}\right\|^{p}>n\right] \\
& =1+2 \sum_{n=1}^{\infty} P\left[\left\|\frac{1}{n} x_{n n}\right\|>1\right]<\infty .
\end{aligned}
$$

Hence,

$$
\mathrm{nP}\left[\left\|\mathrm{x}_{11}\right\|^{\mathrm{p}}>\mathrm{n}\right] \rightarrow 0
$$

By $(2.18)$,

$$
P\left[\left\|\sum_{k=1}^{n} x_{n k}\right\|<n^{1 / p}\right] \rightarrow 1
$$

Therefore, from (2.20) and (2.21) there exists $\mathrm{N}$ such that if $\mathrm{n} \geq \mathrm{N}$ then

$$
\mathrm{nP}\left[\left\|\mathrm{x}_{11}\right\|^{\mathrm{p}}>\mathrm{n}\right]<\frac{1}{4} \text { and } \mathrm{P}\left[\left\|\sum_{\mathrm{k}=1}^{\mathrm{n}} \mathrm{X}_{\mathrm{nk}}\right\|^{\mathrm{P}}<\mathrm{n}\right]>\frac{1}{2} .
$$

Next, define the events

$$
\begin{array}{r}
A_{n k}=\left[\max _{1 \leq i<k}\left\|x_{n i}\right\| \leq 2 n^{1 / p},\left\|x_{n k}\right\|>2 n^{1 / p}, \text { and }\left\|_{\substack{i=1 \\
i \neq k}}^{n} x_{n i}\right\|<n^{1 / p}\right] \\
(k=1,2, \ldots, n: n=1,2, \ldots) .
\end{array}
$$

Clearly, $\left\{A_{n k}: k=1,2, \ldots, n\right\}$ are disjoint subsets of the event $\left[\left\|\sum_{k=1}^{n} x_{n k}\right\|>n^{1 / p}\right]$ for each $n=1,2, \ldots$. A familiar reasoning yields that

$$
\begin{aligned}
& P\left[\left\|\frac{1}{n} \sum_{k=1}^{n} x_{n k}\right\|>1\right] \geq \sum_{k=1}^{n} P\left(A_{n k}\right) \\
& =\sum_{k=1}^{n} P\left[\left\|x_{n k}\right\|>2 n^{1 / p}\right] P\left[\prod_{i=1}^{n-1}\left[\left\|x_{n i}\right\| \leq 2 n^{1 / p}\right] n\left[\left\|\sum_{\substack{i=1 \\
i \neq k}}^{n} x_{n i}\right\|<n^{1 / p}\right]\right] \\
& \geq \sum_{k=1}^{n} P\left[\left\|x_{n k}\right\|>2 n^{1 / p}\right]\left(P\left[\left\|\sum_{i=1}^{n} x_{n i}\right\|<n^{1 / p}\right]-P[\underbrace{n}_{i=1}\left[\left\|x_{n i}\right\|>2 n^{1 / P}\right]]\right) \\
& \geq \sum_{k=1}^{n} P\left[\left\|x_{11}\right\|>2 n^{1 / p}\right]\left(P\left[\left\|\sum_{i=1}^{n-1} x_{n i}\right\|<(n-1)^{1 / P}\right]-n P\left[\left\|x_{11}\right\|>2 n^{1 / p}\right]\right) .
\end{aligned}
$$

Hence, by (2.22), for $n \geq N$,

$$
P\left[\left\|\frac{1}{n} \sum_{k=1}^{n} x_{n k}\right\|>1\right] \geq \frac{1}{4} n P\left[\left\|x_{11}\right\|^{p}>2^{P_{n}}\right] .
$$

Therefore, $\sum_{n=1}^{\infty} \mathrm{nP}\left[\left\|\mathrm{X}_{11}\right\|^{\mathrm{p}}>2^{\mathrm{p}_{\mathrm{n}}}\right]<\infty$. Thus, Lemma 1 yields $\mathrm{E}\left\|\mathrm{X}_{11}\right\|^{2 \mathrm{p}}<\infty$, // 
CONCLUDING REMARKS.

1. It should be noted that the case $\mathrm{p}=1$ in Theorem 6 is obtainable in a type 1 space (cf: Theorem 4 of $\mathrm{Hu}$, Moricz and Taylor [5]). In which case type $1+\delta$ is not needed.

2. For sequences of independent random elements which are uniformly bounded by a random variable $\mathrm{X}$ with $\mathrm{E}|\mathrm{X}|^{\mathrm{p}}<\infty$, (1.6) holding necessitates the space being of type $p+\delta$ (cf: Woycyznski [4] and Maurey and Pisier [8]). Thus, the necessity of type $p+\delta$ follows for Theorem 4 .

3. Theorem 6 shows that Theorem 4 is the best possible moment condition when no conditions on possible relations between the rows of the array are assumed.

4. In [4] it is mentioned that $n^{-1 / p} \sum_{k=1}^{n} x_{k} \rightarrow 0$ a.s. for i.i.d. random elements $\left\{X_{n}\right\}$ with $\mathrm{EX}_{1}=0$ and $E\left\|X_{1}\right\|^{p}<\infty$ apparently is equivalent to the space being of type p. Thus, it is interesting to conjecture whether Theorem 6 remains valid for only type $p$ spaces $1 \leq p<2$. Certainly, the "if part" is true for type $p$ spaces, and Remark 1 indicates that it is true $p=1$.

ACKNOWLEDGEMENTS. This research was supported in part by the Air Force Office of Scientific Research under Contract No. F49620 85 C 0144 while the first named author was at the Center for Stochastic Processes, University of North Carolina, Chapel Hill, N.C. The research for the second named author was mainly completed while at the Department of Statistics, University of Georgia, Athens, GA.

\section{REFERENCES}

1. HSU, P.L., and ROBBINS, H. (1947). Complete Convergence and the Law of Large Numbers. Proc. Nat. Acad. Sci. U.S.A., 33, 25-31.

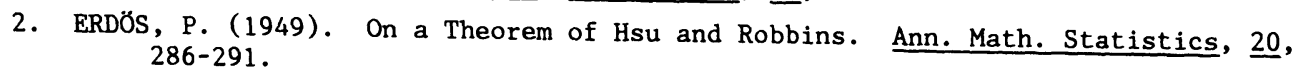

3. JAIN, N.C. (1975). Tail Probabilities for Sums of Independent Banach Space Random Variables. Z. Wahr. v. Geb., 33, 155-166.

4. WOYCZYNSKI, W.A. (1980). On Marcinkiewicz-Zygmund Laws of Large Numbers in Banach Spaces and Related Rates of Convergence. Prob. and Math. Statist., 1, 117-131.

5. HU, T.C, MÓRICZ, F.and TAYLOR, R.L. (1986). Strong Laws of Large Numbers for Arrays of Rowwise Independent Random Variables. Statistics Technical Report 27, University of Georgia, March, 1986.

6. BECK, A. (1963). On the Strong Law of Large Numbers. Ergodic Theory. Academic Press, New York, 21-53.

7. TAYLOR, R.L. (1982). Convergence of Weighted Sums of Arrays of Random Elements in Type p Spaces with Application to Density Estimation. Sankhya, 44, 341-351.

8. MAUREY, B. and PISIER, G. (1976). Series des Variables Aléatoires Vectorielles Independantes et Proprietés Geométriques des Espaces de Banach. Studia Math.,
58, 45-90. 


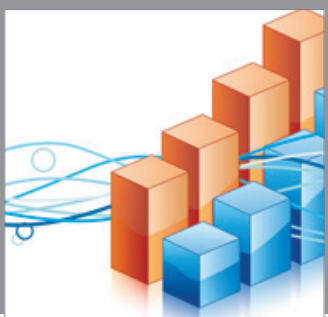

Advances in

Operations Research

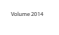

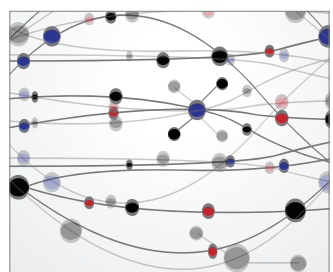

\section{The Scientific} World Journal
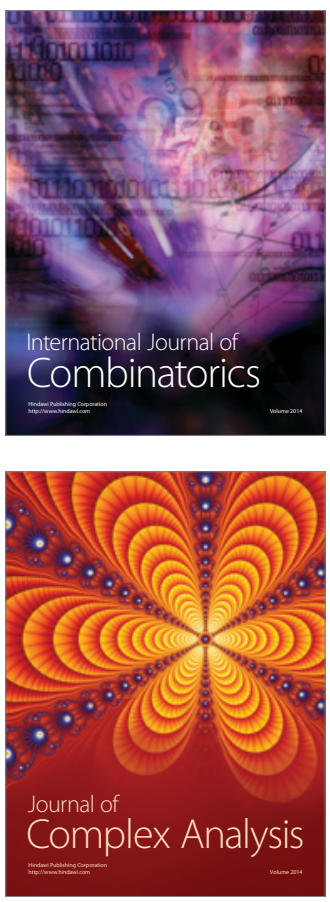

International Journal of

Mathematics and

Mathematical

Sciences
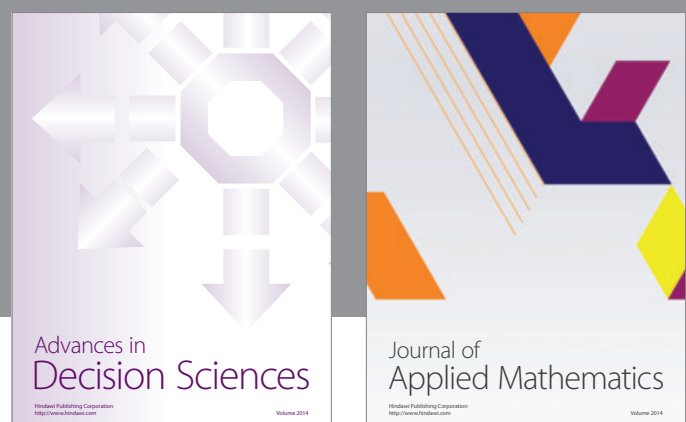

Journal of

Applied Mathematics
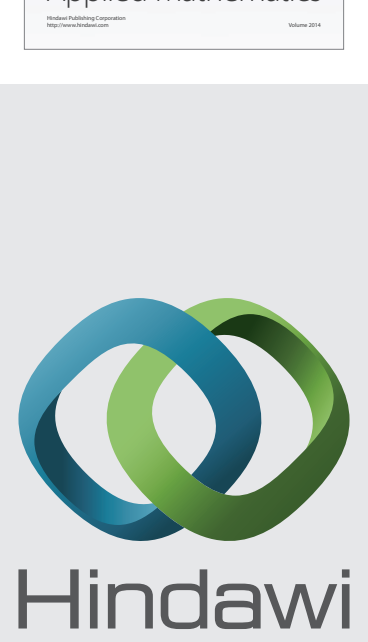

Submit your manuscripts at http://www.hindawi.com
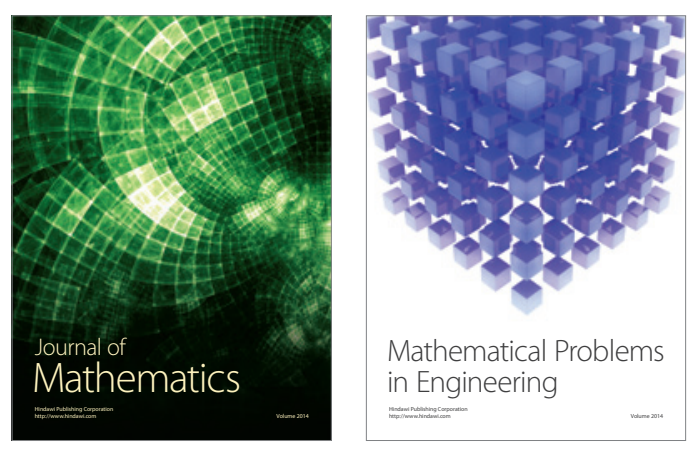

Mathematical Problems in Engineering
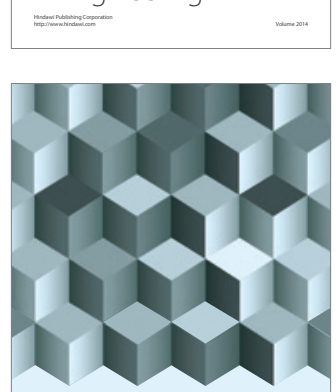

Journal of

Function Spaces
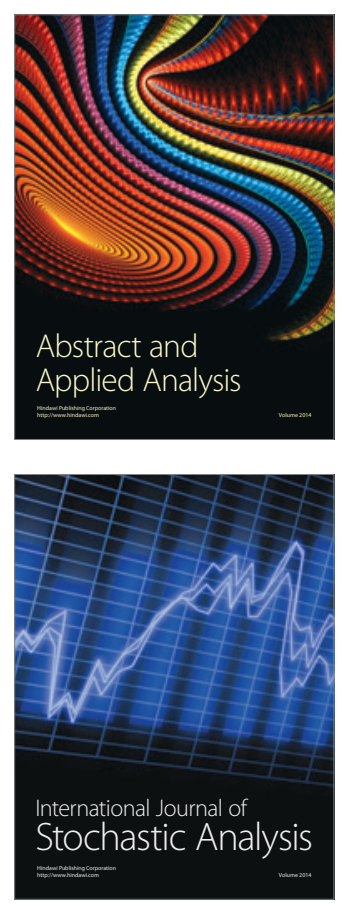

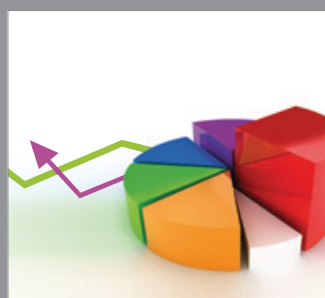

ournal of

Probability and Statistics

Promensencen
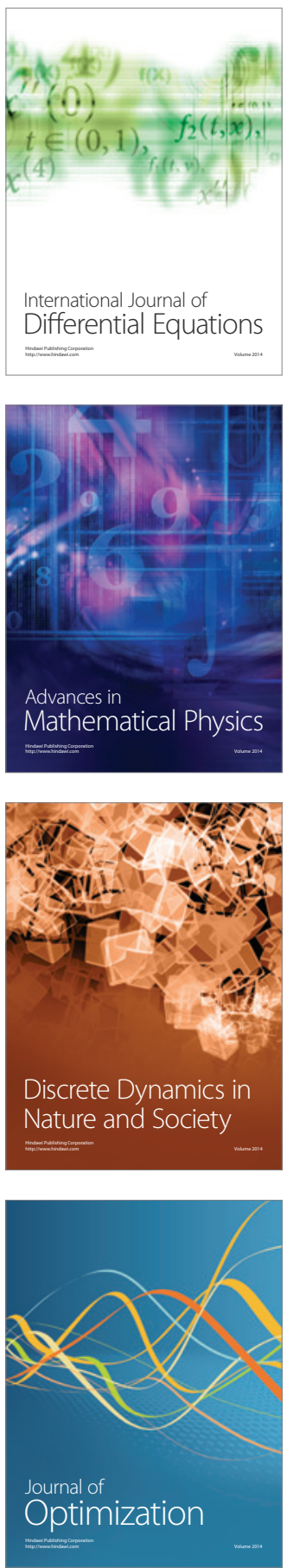